\title{
STUDY OF A FAR INFRARED CAVITY AROUND AN AGB STAR UNDER IRAS SURVEY AT GALACTIC LATITUDE - $1.6^{\circ}$
}

\section{A. K. Gautam and B. Aryal}

Journal of Nepal Physical Society

Volume 5, Issue 1, October 2019

ISSN: $2392-473 X$

Editors:

Dr. Vinaya Kumar Jha

Dr. Binod Adhikari

Dr. Kapil Adhikari

JNPS, 5 (1), 35-41 (2019)

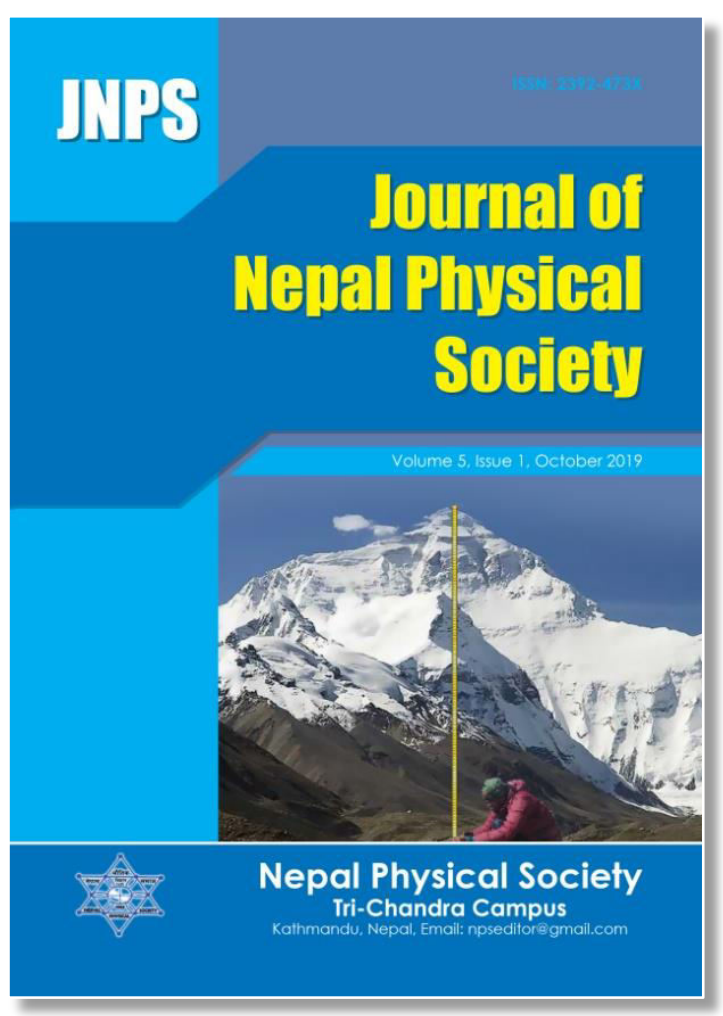

Published by:

Nepal Physical Society

P.O. Box: 2934

Tri-Chandra Campus

Kathmandu, Nepal

Email:npseditor@gmail.com 


\title{
STUDY OF A FAR INFRARED CAVITY AROUND AN AGB STAR UNDER IRAS SURVEY AT GALACTIC LATITUDE -1.6
}

\author{
A. K. Gautam ${ }^{1, *}$ and B. Aryal ${ }^{2}$ \\ ${ }^{1}$ Bhaktapur Multiple Campus, T.U., Bhaktapur, Nepal \\ ${ }^{2}$ Central Department of Physics, T.U., Kirtipur, Nepal \\ Corresponding Email: arjungautamnpj@gmail.com, baryal@tucdp.edu.np
}

\begin{abstract}
In this paper, we discuss about the physical properties of the dusty environment around the AGB star located

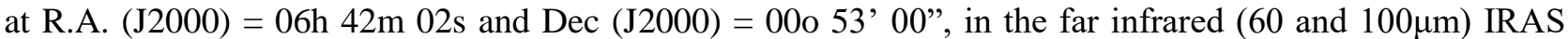
maps. A cavity like structure (major diameter $\sim 2.5 \mathrm{pc} \&$ minor diameter $\sim 0.9 \mathrm{pc}$ ) is found to lie at R.A. $(\mathrm{J} 2000)=06 \mathrm{~h} 41 \mathrm{~m} 43.03 \mathrm{~s}$ and DEC $(\mathrm{J} 2000)=01 \mathrm{o} 09^{\prime} 22.8^{\prime \prime}$, located at a distance $\sim 405 \mathrm{pc}$ from the star. By using contour map diagram, we studied the distribution of flux density, dust color temperature, dust mass and outflow mass in the cavity. The dust color temperature is found to lie in the range $21.4 \mathrm{~K}$ to $21.9 \mathrm{~K}$ which shows the cavity is isolated and independently evolved. Such a low offset temperature variation shows that the star is stable and its life is long. Dust particles are less interacting and mean free path is large. The cavity may be in thermally pulsating phase. Product of dust color temperature and visual extinction is found to be less than one. A possible explanation of the results will be discussed.
\end{abstract}

Key Words: AGB Stars, dust temperature, dust mass, dredge-up process, s-process, excess mass

\section{INTRODUCTION}

The AGB phase is particularly important to understand the origin of various elements heavier than $\mathrm{H}$ and $\mathrm{He}$, and how they influence the chemical evolution of their hosting systems. AGB stars are also the major contributors of the integrated light of stellar systems of intermediate age and, therefore, important tools to study extragalactic systems [1]. The AGB is populated by evolving stars of low and intermediate mass. As mentioned above, the limits of low mass stars are defined by the development of an electron degenerate core after leaving the MS. Intermediate stars ignite their He-cores under non-degenerate conditions. The AGB phase of low-mass stars and intermediate mass stars up to $8 \mathrm{M}_{\odot}$ is characterized by an electron degenerate core of $\mathrm{O}$ and $\mathrm{C}$, which is surrounded by two thin shells in which nuclear $\mathrm{H}$ and He-burning occurs.

Two phases in the AGB star are usually distinguished: the early AGB (EAGB) phase and the phase, in which the stars start to thermally pulse (TP-AGB phase). The early AGB (E-AGB) phase begins as soon as the star finishes burning $\mathrm{He}$ in its core and begins to burn $\mathrm{He}$ in a thick shell. When both $\mathrm{H}$ and $\mathrm{He}$ burning shells are active, the star enters the thermally pulsating AGB (TP-AGB) phase [2].

The AGB phase is characterized by a strong massloss period that only stops when the outer envelope is completely lost [3]. As the star ascends the AGB, the massloss rate increases from solar-like values $\left(10^{-14} \mathrm{M}_{\odot} / \mathrm{yr}\right)$ up to $10^{-4} \mathrm{M}_{\odot} / \mathrm{yr}$, the so-called super-wind phase. Dust grains and molecules, predominantly $\mathrm{CO}$, are formed in the wind, producing a circumstellar envelope detectable in the infrared and millimetric domains. Although the radiation pressure on dust grains and on molecules is thought to play an important role in the driving mechanism of the wind, different physical aspects governing the dust formation/destruction processes are still unclear. Processed elements are dredged-up into the atmosphere as a consequence of the re-ignition of the He burning shell (E-AGB phase). A further contraction leads also to the re-ignition of the $\mathrm{H}$ burning shell and the star enters a TP-AGB phase. A third dredge-up may take place during this phase and overshooting will contribute to lift carbon to the surface. These events lead to the formation of circumstellar envelopes whose chemical composition reflects that of an atmosphere enriched with nuclear processed 
matter. C-rich stars have envelopes composed mainly of amorphous carbon while O-rich stars have circumstellar envelopes rich in silicates. Both oxidic and carbonaceous dust particles are characterized by vibrational bands positioned in the mid-IR window (at $9.7 \mu \mathrm{m}$ and $18 \mu \mathrm{m}$ for silicates and at $11.3 \mu \mathrm{m}$ for $\mathrm{SiC}$ ) [4]. Most of the thermal radiation continuum from the dusty envelope is emitted in this wavelength range.

AGB stars are very complex objects characterised by a variety of different processes that take place at different locations within the star. Compared to the small cores, AGB stars have huge atmospheres of low density and low temperature and, therefore, exhibit no well-defined boundaries. Furthermore, they are characterised by dynamical processes as pulsation, shock waves, dust formation and mass loss. The spectral features are significantly different for stars with O-rich and C-rich atmospheres.
Initially, the abundance ratio of $n(\mathrm{C})$ over $n(\mathrm{O})$ is smaller than one $(\mathrm{C} / \mathrm{O}<1)$ and most of the stars can be classified as O-rich AGB stars of spectral type M. For AGB stars with initial masses below $4 M$ the atmospheric chemical composition can change dramatically since processed elements, most notably $12 \mathrm{C}$, are dredged up to the surface by convective mixing after a thermal pulse. Depending on the C/O-ratio their spectral type changes from $\mathrm{K}$ or $\mathrm{M}$ via $\mathrm{S}$ to $\mathrm{C}(\mathrm{C} / \mathrm{O}>1, \mathrm{C}$-rich $\mathrm{AGB}$ star.

\section{METHODS}

We investigated a cavity-like structure in both 60 and 100 micron IRAS maps around a AGB star. We briefly describe a method for calculation of dust color temperature and dust mass of the dusty environment around carbon-rich Asymptotic Giant Branch named AGB06+00.
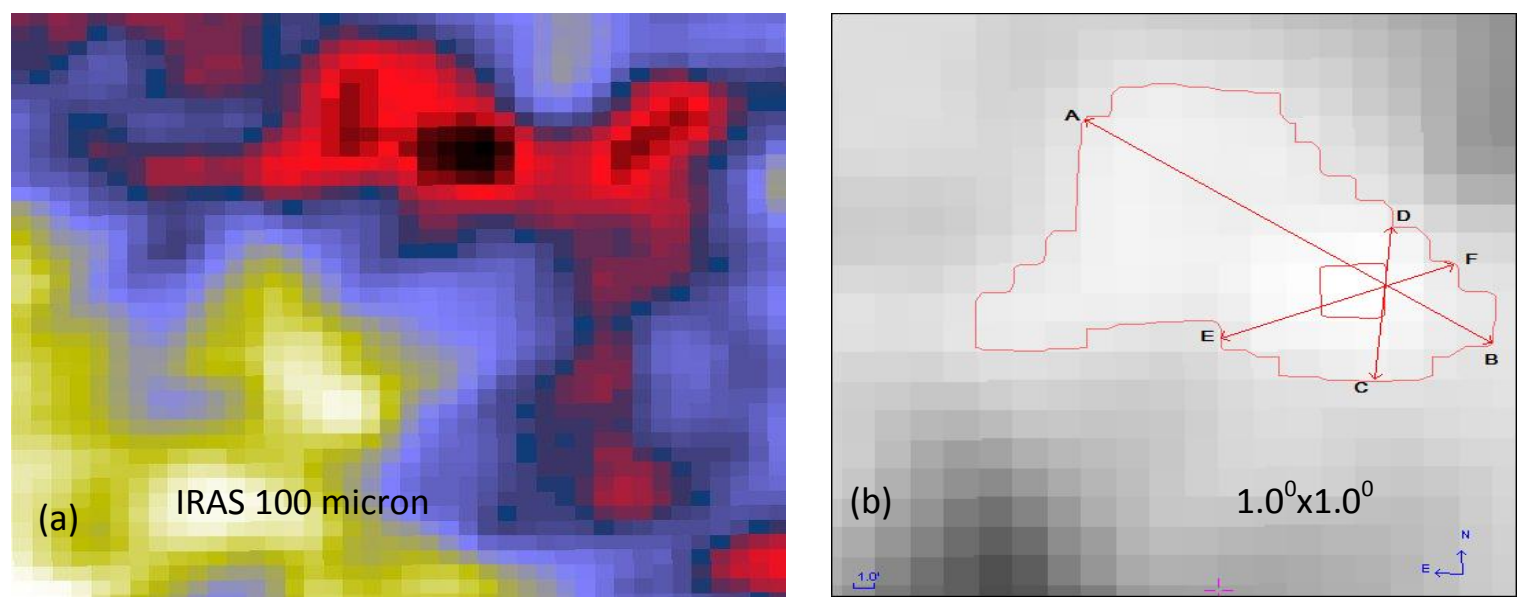

Fig. 1: (a) IRAS $100 \mu \mathrm{m}$ and $60 \mu \mathrm{m}$ far infrared image of the core region of AGB 06+00 centered at R.A. $(\mathrm{J} 2000)=06^{h} 41^{m} 43.04^{s}$, Dec. $(\mathrm{J} 2000)=01^{0} 09^{\prime} 22.8^{\prime \prime}$ and (b) Contour map of the cavity where major diameter $(A B)$, minor diameter $(C D)$ and diameter between minimum flux \& minimum temperature.

\subsection{Dust Color Temperature Estimation}

IRAS works on four bands i.e., at 12, 25, 60 and $100 \mu \mathrm{m}$. Schnee et al. [5] calculated dust color temperature by using $60 \mu \mathrm{m}$ and $100 \mu \mathrm{m}$ IRAS maps. Flux density is the product of Planck function and opacity. From modified power law, the flux density of emission at a wavelength $\lambda_{\mathrm{i}}$ is given by

$\mathrm{F}_{\mathrm{i}}=\left[\frac{2 h c}{\lambda_{i}^{3}\left(e^{\frac{h c}{\lambda_{i} k T_{d}}}\right)-1}\right] \mathrm{N}_{\mathrm{d}} \alpha \lambda_{\mathrm{i}}^{-\beta} \Omega_{i}$ where $\beta$ is the spectral emissivity index, $N_{d}$ is the column density of dust grains, $\alpha$ is a constant i.e. free parameter which relates the flux with the optical depth of the dust, and $\Omega_{\mathrm{i}}$ is the solid angle subtended at $\lambda_{\mathrm{i}}$ by the detector. In Dupac et al. [6], there is an inverse relationship between temperature and emissivity spectral index.

Since the dust emission is optically thin at $60 \mu \mathrm{m}$ and $100 \mu \mathrm{m}$ and that $\Omega_{60} \sim \Omega_{100}$ (true for IRAS image), we can write the ratio, $R$, of the flux densities at $60 \mu \mathrm{m}$ and $100 \mu \mathrm{m}$ as

$\mathrm{R}=0.6^{-(3+\beta)} \frac{e^{144 / T_{d}}-1}{e^{240 / T_{d}}-1}$ 
The spectral emissivity index $(\beta)$ depends on dust grain properties like composition, size, and compactness. For a pure blackbody would have $\beta=$ 0 , the amorphous layer-lattice matter has $\beta \sim 1$, and the metals and crystalline dielectrics have $\beta \sim 2$ which is used in our calculations.

For a smaller value of $T_{d}, 1$ can be dropped from both numerator and denominator of Eq. (3) and it takes the form

$\mathrm{R}=0.6^{-(3+\beta)} \frac{e^{144 / T_{d}}}{e^{240 / T_{d}}} \cdots$

where, $R=\frac{F(60 \mu \mathrm{m})}{F(100 \mu \mathrm{m})}$

Taking natural logarithm on both sides of Eq. (4) and solving it, we find the expression for the temperature as

$$
\begin{aligned}
& \ln (\mathrm{R})=\ln 0.6^{-(3+\beta)}\left[144 / \mathrm{T}_{\mathrm{d}}-240 / \mathrm{T}_{\mathrm{d}}\right] \\
& =\ln 0.6^{-(3+\beta)}\left[-96 / T_{d}\right] \\
& \mathrm{T}_{\mathrm{d}}=\frac{-96}{\ln \left\{\mathrm{R} \times 0.6^{(3+\beta)}\right\}}
\end{aligned}
$$

$\mathrm{F}(60 \mu \mathrm{m})$ and $\mathrm{F}(100 \mu \mathrm{m})$ are the flux densities in $60 \mu \mathrm{m}$ and $100 \mu \mathrm{m}$ respectively and Eq. (5) can be used for calculation of the dust grain temperature.

\section{Dust Mass Estimation}

Dust mass is another important physical quantity which is useful to analysis the cavity structure. Hildebrand has calculated dust mass at $100 \mu \mathrm{m}$ wave length. For it known distance of the cavity from the observing region is needed. The known distance of the loops from observing region was provided in catalog of far infrared loops in the galaxy [7].

For the calculation of dust mass, we first obtained the value of flux density $\left(S_{v}\right)$ at $100 \mu \mathrm{m}$ maps. The dust mass is estimated using [8],

$\mathrm{M}_{\text {dust }}=\frac{4 \mathrm{ar}}{3 \mathrm{Q}_{\mathrm{n}}}\left|\frac{\mathrm{S}_{\mathrm{n}} \mathrm{D}^{2}}{\mathrm{~B}(\mathrm{n}, \mathrm{T})}\right|$

where, weighted grain size (a) $=0.1 \mu \mathrm{m}$, grain density $(\rho)=3000 \mathrm{~kg} \mathrm{~m}^{-3}$, grain emissivity $\left(\mathrm{Q}_{v}\right)=$ 0.0010 (for $100 \mu \mathrm{m}$ ) [10].

The Planck function $\mathrm{B}(v, T)$, which is the function of temperature and frequency and is given by the expression:
$\mathrm{B}(v, \mathrm{~T})=\frac{2 \mathrm{hc}}{\mathrm{l}^{3}}\left(\frac{1}{\frac{\mathrm{hc}}{\mathrm{e}^{\mathrm{lkT}}-1}}\right)$

is the Planck function.

where, $\mathrm{h}=$ Planck's constant, $\mathrm{c}=$ velocity of light, $v=$ frequency at which the emission is observed, $\mathrm{T}$ $=$ the average temperature of the region.

For $100 \mu \mathrm{m}$ wavelength, the expression (7) for the dust mass reduces to,

$\mathrm{M}_{\text {dust }}=0.4\left[\frac{\mathrm{S}_{\mathrm{n}} \mathrm{D}^{2}}{\mathrm{~B}(\mathrm{n}, \mathrm{T})}\right]$.

We use equation (9) to calculate dust mass of the cavity.

\section{Estimation of Visual Extinction}

For estimation of visual extinction, Wood et al. [9] has provided an empirical formula. According to them, we have

$$
A_{\mathrm{V}}(\mathrm{mag})=15.078\left(1-\exp \left(-\tau_{100} / 641.3\right)\right)
$$

Where

$$
\tau_{100}=\frac{F_{\lambda}(100 \mu m)}{B_{\lambda}\left(100 \mu m, T_{d}\right)}
$$

is optical depth at $100 \mu \mathrm{m}$ wavelength. Here $F_{\lambda}$ is flux density and $B_{\lambda}$ is Planck function at $100 \mu \mathrm{m}$ wavelength.

\section{RESULT AND DISCUSSION \\ Structure: Contour Maps}

While going through the systematic search on IRAS maps, we discovered an isolated cavity in the 100 $\mu \mathrm{m}$ and $60 \mu \mathrm{m}$ at R.A. (J2000) $=06^{\mathrm{h}} 41^{\mathrm{m}} 43.04^{\mathrm{s}}$ and Dec. $(\mathrm{J} 2000)=01^{\circ} 09^{\prime} 22.8^{\prime \prime}$. With the help of the software ALADIN2.5, we have studied physical properties (size, dust color temperature, dust mass, etc) of the cavity. We selected contour level in such a way that it circles the cavity. The major axis, minor axis and line passing through minimum temperature and minimum flux are shown in the fig.1(b).

\section{Distribution of Flux Density}

By using ALADIN 2.5 software, the values of flux densities at $60 \mu \mathrm{m}$ and $100 \mu \mathrm{m}$ have measured. The flux density distribution within the contour of the region of interest has studied. We plotted a graph between flux at $100 \mu \mathrm{m}$ and $60 \mu \mathrm{m}$ with the help of 
ORIGIN 5.0 which is shown in fig.2(a). From the linear fit, slope of the line was 0.21 . The linear equation of the fitted line is, $y=-1.3+0.2 x$. Using

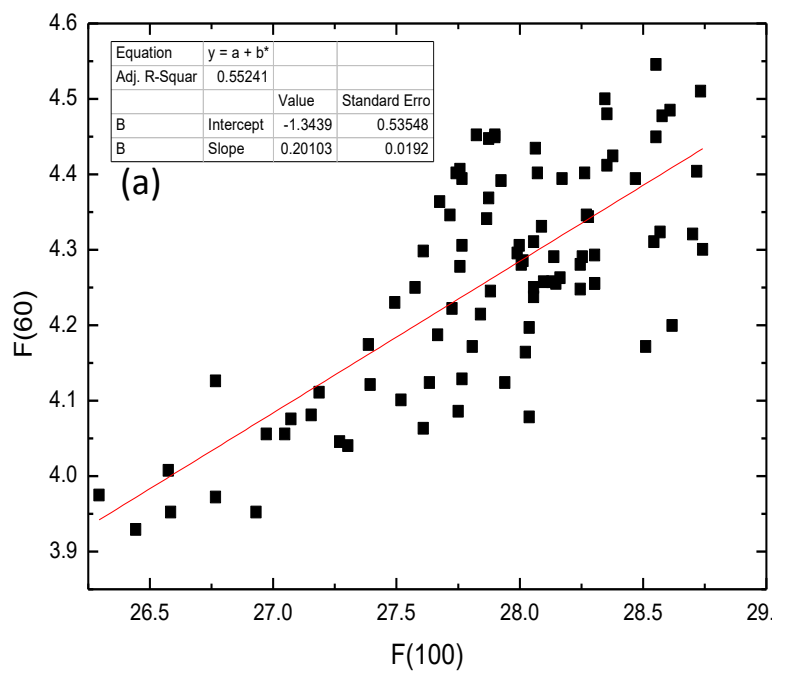

the slope of best fitted plot, dust color temperature is found as $23.1 \mathrm{~K}$ which is nearly similar with our calculated value.

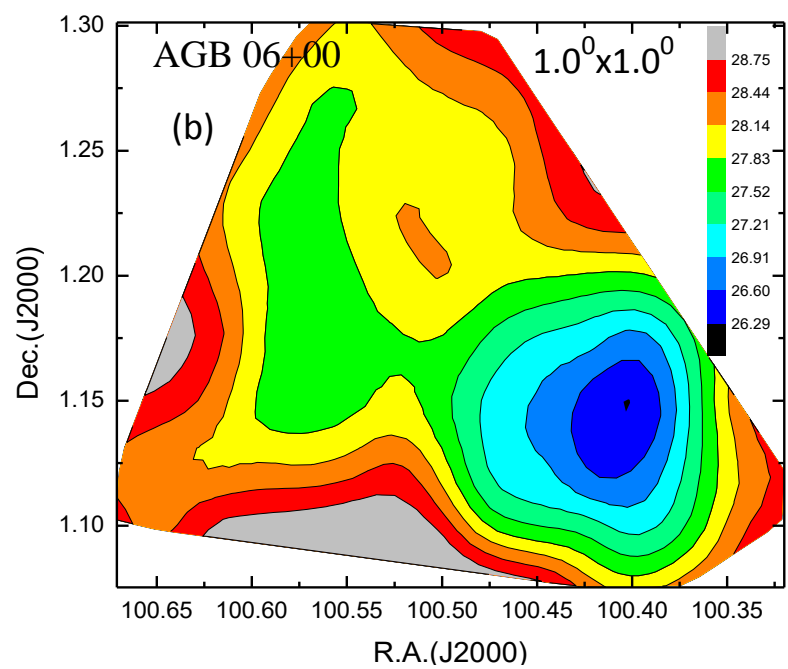

Fig. 2 : (a) The $100 \mu \mathrm{m}$ verses $60 \mu \mathrm{m}$ flux density in the region of interest and (b) Contour map at 100 $\mu \mathrm{m}$ flux

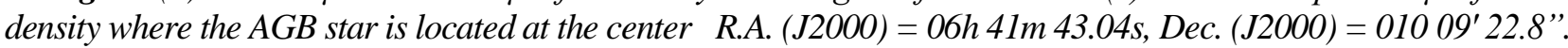

Again distribution of flux at $100 \mu \mathrm{m}$ of the pixels within the contour level with right ascension (R.A.) and declination (Dec.) are plotted by using ORIGIN 8.0 and the graph is shown in fig.2(b). Graph shows that all the fluxes from minimum to maximum lie within the contour level. Most of the maximum flux regions lie at the boundary.

\section{Dust color Temperature and its Variation}

Using the method of [5], we calculated dust color temperature of each pixel inner the outer isocontour in the region of interest. We use the IRAS $100 \mu \mathrm{m}$

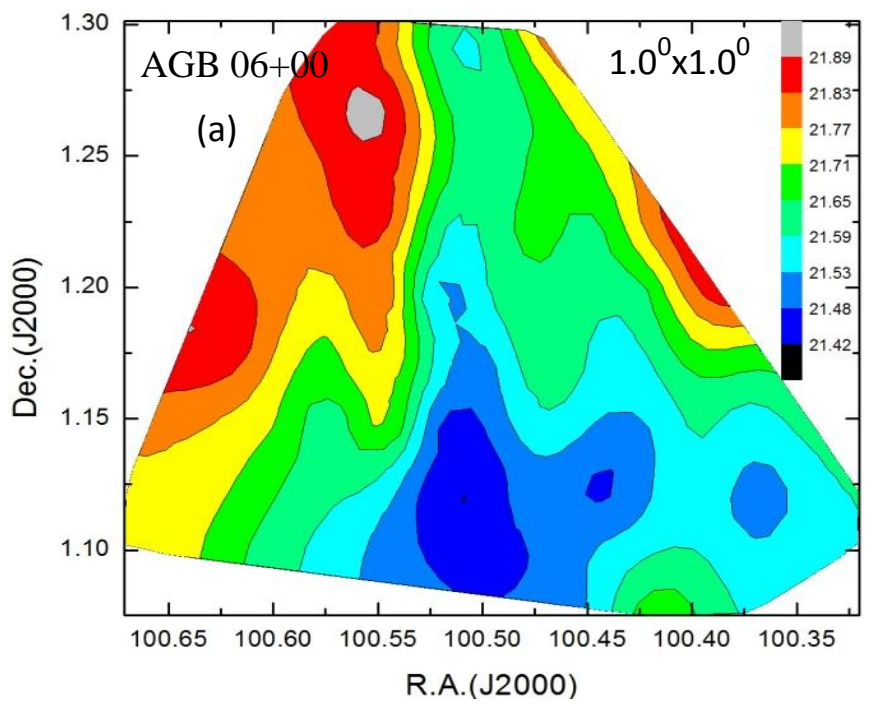

and $60 \mu \mathrm{m}$ FITS images downloaded from the IRAS server . For the calculation of temperature we choose the value of $\beta=2$ following the explanation given by (6). Variation of temperature with corresponding R.A.(J2000) and Dec.(J2000) are plotted by using ORIGIN 8.0 and the graph is shown in figure 3(a). Graph shows that temperature distributions are in separate cluster but minimum temperature region is little bit shifted from minimum flux density which is unusual behavior. Such type of nature is obtained due to external factors.

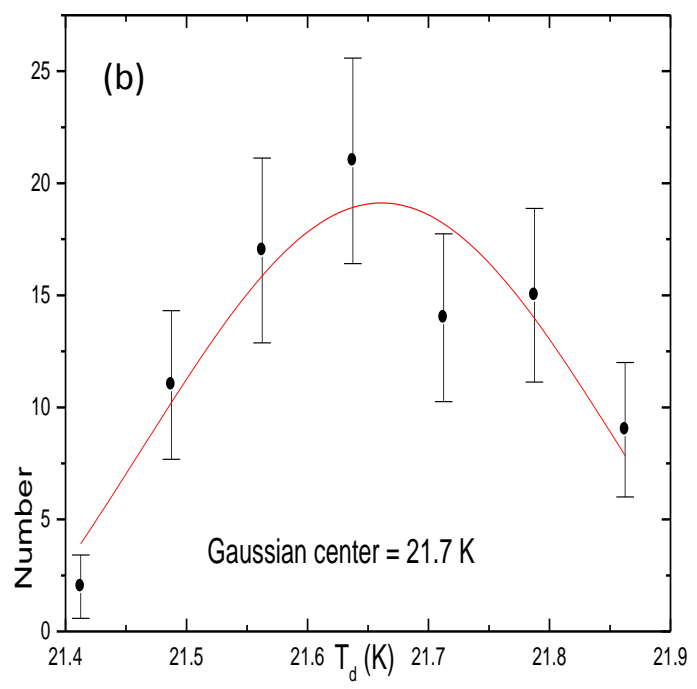

Fig. 3: (a) Contour map of dust color temperature and (b) Gaussian fit between dust color temperature and number of pixels. The field is centered at center R.A. $(J 2000)=06^{h} 41^{m} 43.04^{s}$, Dec. $(J 2000)=01^{0} 09^{\prime} 22.8^{\prime \prime}$. 
The region in which minimum and maximum temperature is found in the range of $21.4 \mathrm{~K}$ to $21.9 \mathrm{~K}$ with an offset temperature of dust $0.5 \mathrm{~K}$. Such a low offset temperature variation shows that there is symmetric outflow or symmetric distribution of density and temperature. When this result is compared with the result obtained in [11] where temperature variation is $20 \mathrm{~K}$ to $22 \mathrm{~K}$ so our result is also comparable with that result. In the contour map, minimum flux and minimum temperature region are shifted which is due to some external factors possibly due to AGB wind. There is good agreement in case of temperature in the Gaussian fit with offset $-8.7 \mathrm{~K}$.

\section{Size of the Structure}

Major and minor diameter of the structure can be easily calculated by using a simple expression i.e., $\mathrm{L}=$

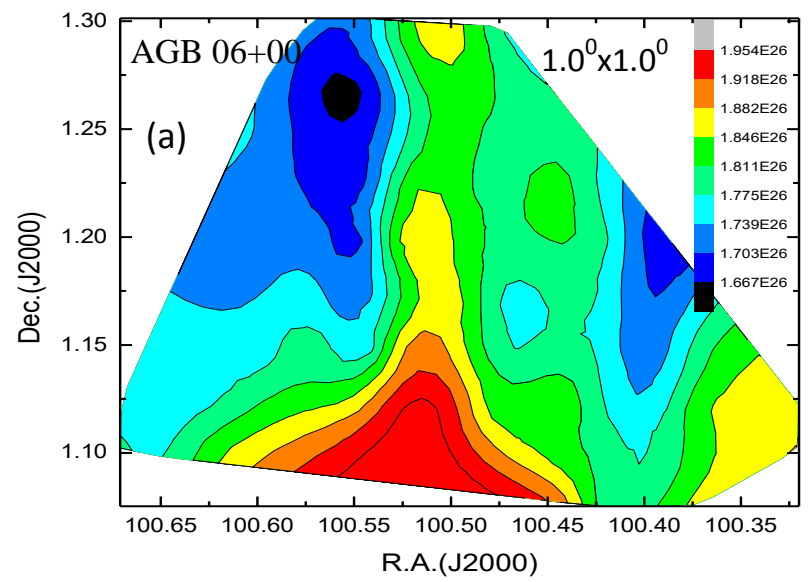

$\mathrm{R} \times \theta$, where $\mathrm{R}=405 \mathrm{pc}$ [7] is the distance of the structure and $\theta=$ pixel size (in radian). After calculation the major and minor diameter of the cavity region are found to be $2.5 \mathrm{pc}$ and $0.9 \mathrm{pc}$ respectively. Thus, the size of the structure is $2.5 \mathrm{pc} \times 0.9 \mathrm{pc}$.

\section{Dust Mass Estimation and its variation}

For the calculation of dust mass, we need the distance to the region of interest. The distance of the structure is $405 \mathrm{pc}$ [7]. By using the temperature of each pixel and corresponding distance of the structure, we calculated mass of each pixel. Average mass of each pixel is $1.8 \times 10^{26} \mathrm{~kg}$ and total mass of the structure is $1.6 \times 10^{28} \mathrm{~kg}$ i.e $0.008 \mathrm{M}_{\odot}$. But mass of dust obtained around white dwarf WD $1003-44$ in [10] is $0.08 \mathrm{M}_{\odot}$. It means mass of dust around AGB Star is less than White Dwarf.

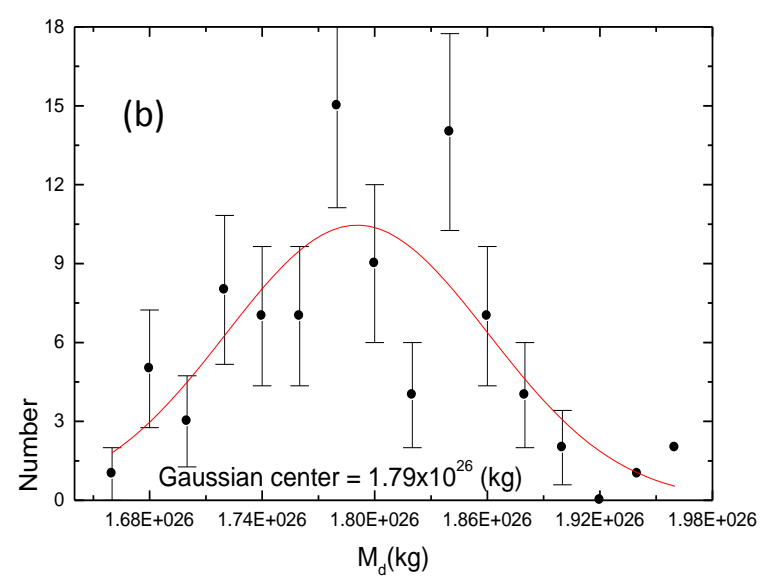

Fig.4: (a) Contour map of dust mass and (b) Gaussian fit between mass and number of pixels. The field is centered at R.A. $(\mathrm{J} 2000)=06^{h} 41^{m} 43.04^{s}$, Dec. $(J 2000)=01^{0} 09^{\prime} 22.8^{\prime \prime}$.

Distribution of dust mass of the pixels within the selected contour level with R.A. (J2000) and Dec.(J2000) are plotted in contour map by using ORIGIN 8.0. Graph obtained is shown in fig.4(a) which shows that minimum mass region lie at the maximum temperature region in the selected contour which is usual trend. There is no good agreement in case of dust mass where offset mass is $-0.02 \mathrm{~kg}$.

\section{Variation of Visual Extinction}
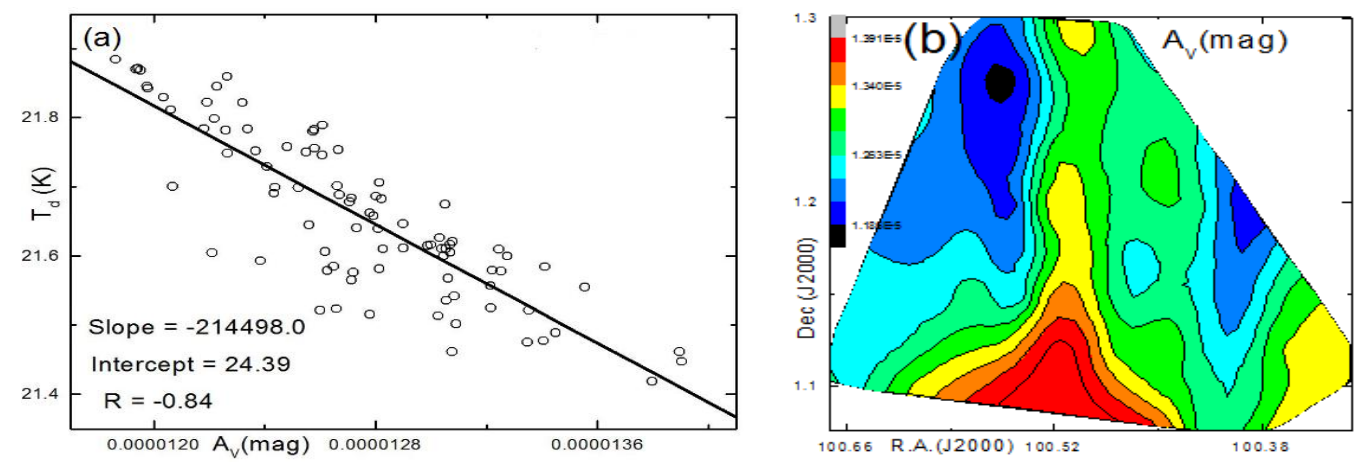

Fig. 5: (a) Variation of visual extinction with dust color temperature and (b) Contour map of visual extinction. The field is centered at R.A. $(J 2000)=06^{h} 41^{m} 43.04^{s}$, Dec. $(J 2000)=01^{0} 09^{\prime} 22.8^{\prime \prime}$. 
Figure 5(a) is the linear fit between visual extinction and dust color temperature which shows a systematic trend between them with high correlation coefficient. From the graph, it is found that $A_{V} X T_{d}<1$ i.e., higher the temperature, lower the visual extinction and vice-versa. Figure 5(b) is the contour map of visual extinction which is exactly same with contour map of dust mass. It means they have linear relation. When we compare it with contour map of dust color temperature, we get higher the visual extinction, lower the temperature and vice-versa. It means relation between visual extinction and dust color

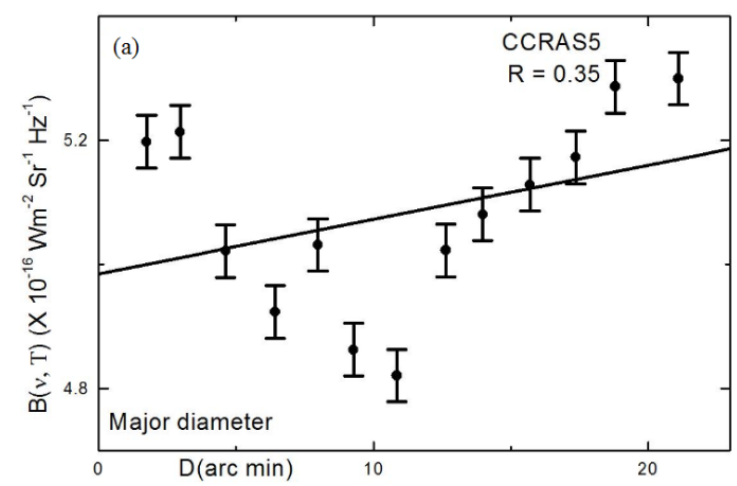

temperature obtained from figure 5(a) is justified by their contour maps.

\section{Variation of Planck function with distance}

Figure 6 shows the variation of Planck function along extension and compression where extension corresponds to major diameter and compression corresponds to minor diameter. Planck function at the particular point along both diameters are measured and they are plotted with the best fitted line where random distribution is found means systematic trend could not be obtained along both diameter. It means dust particles along the diameters are not in thermal equilibrium.

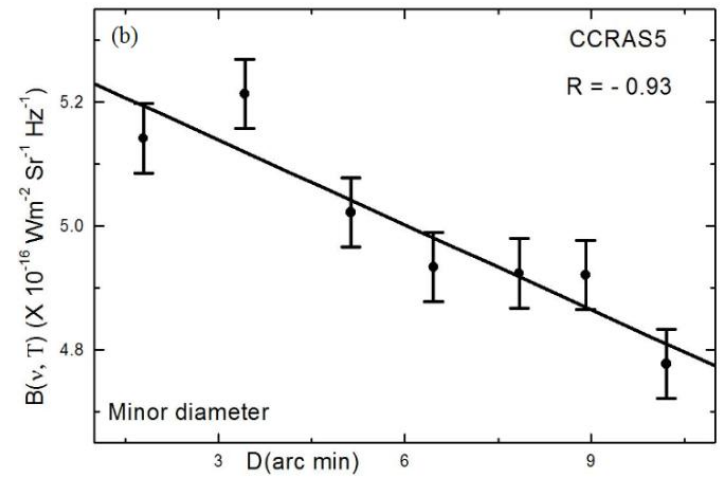

Fig.6: Variation of Planck function $B(v, T)$ along major diameter (a) and minor diameter

(b) passing through minima of the cavity around the AGB star namely AGBO6+OO centered at R.A. $(J 2000)=06^{h} 41^{m} 43.04^{s}$, Dec. $(J 2000)=01^{0} 09^{\prime} 22.8^{\prime \prime}$.

\section{Calculation of Excess Mass}

For the calculation of excess mass, we have drawn two circles i.e. inner and outer circle with the help of software Aladin V8.0. Circle through major diameter is supposed as outer circle and the circle through minor diameter is supposed as inner diameter of the interested region as shown in figure 7 . With the help of those circles we have calculated excess mass.

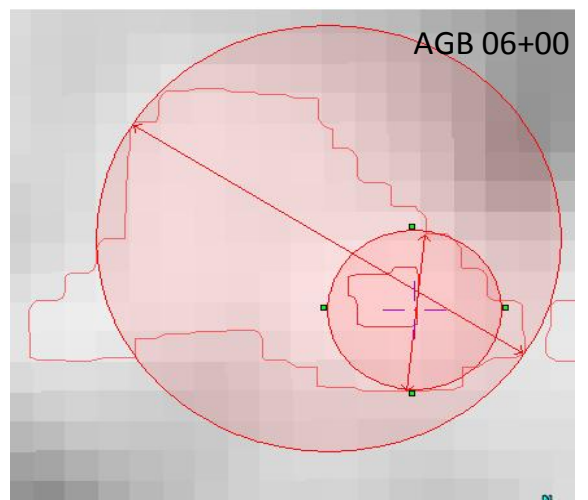

Fig. 7: The inner and outer circle drawn in the structure of the cavity around the AGBO6+00 centered at R.A. $(J 2000)=06^{h} 41^{m} 43.04^{s}$, Dec. $(J 2000)=01^{0}$ $09^{\prime} 22.8$ " for calculation of excess mass.
From the calculation total mass of the inner circle was found to be $4.0 \times 10^{27} \mathrm{~kg}$ and the total mass of outer circle including inner circle was $2.9 \times 10^{28} \mathrm{~kg}$. So the mass deficit in the inner pixel which was blown away by the AGB star is $2.5 \times 10^{28} \mathrm{~kg}$ i.e. $0.01 \mathrm{M}_{\odot}$

\section{CONCLUSION}

The physical properties of the cavity-like structure that we investigated while searching a effect of AGB wind around carbon-rich AGB stars. A study of flux density and dust color temperature maps mass of dust, mass deficit of the cavity was calculated. Our conclusions are as follows:

- The major and minor diameter of the cavity like structure was found to be $2.5 \mathrm{pc}$ and $0.9 \mathrm{pc}$ respectively.

- The minimum temperature $21.4 \mathrm{~K}$ was found at R.A. $(\mathrm{J} 2000)=100.5^{0} \&$ Dec. $(\mathrm{J} 2000)=1.1^{0}$ and maximum temperature $21.9 \mathrm{~K}$ was found at R.A. $(\mathrm{J} 2000)=100.6^{0} \&$ Dec. $(\mathrm{J} 2000)=1.3^{0}$ with offset of $0.5 \mathrm{~K}$.. Low offset in the temperature showed that there is symmetric outflow or 
symmetric distribution of density and temperature so that the entire cavity is stable.

- In general, minimum flux and minimum temperature lie at same point in the pixel but in this case minimum temperature is shifted which may be due to external factors, possibly due to wind emitted from the AGB star. Similarly maximum temperature and minimum mass region lie at same region which is normal behavior.

- Average mass of dust in each pixel is $1.8 \times 10^{26}$ $\mathrm{kg}$.

- Contour map of dust color temperature and dust mass shows that minimum temperature region is the heaviest which support the cosmological principle i.e., distribution of mass is homogeneous and isotropic.

- Gaussian fit are used to study about their symmetry but this case both dust color temperature and dust mass did not show Gaussian nature.

- Total mass of inner circled cavity was $4.0 \times 10^{27}$ $\mathrm{Kg}$ and that of the outer circle including inner circle was $2.9 \times 10^{28} \mathrm{~kg}$. The mass deficit of the structure was $2.5 \times 10^{28} \mathrm{~kg}$ i.e. $0.01 \mathrm{M}_{\odot}$.

- From the linear fit between visual extinction and dust color temperature, we found $\mathrm{A}_{\mathrm{V}} \mathrm{X} \mathrm{T}_{\mathrm{d}}<1$ i.e., higher the temperature, lower the visual extinction and vice-versa which is agreed by their contour map.

- Variation of Planck function along both diameters showed that dust particles along the diameters are in random motion and are not in thermal equilibrium.
We intend to study the role of cabon-rich AGB star to form the far-infrared cavity in the future.

\section{ACKNOWLEDGEMENTS}

We are grateful to the Department of Astro-Particle Physics, Innsbrck University, specially to the team of Prof. R. Weinberger for invoking us to work on dusty environments around AGB stars. This research has made use of SkyView Virtual Observatory, Aladin v2.5 and NASA/IPAC Extragalactic Database (NED).

\section{REFERENCES}

[1] P. Battinelli \& S. Demers, A\&A, 417, 479 (2004).

[2] I. Iben, \& A. Renzini, ARA\&A, 21, 271 (1983).

[3] H.J Habing, A\&A Rev., 7, 97 (1996).

[4] T. Henning, IAU Symp.191,221 (1999).

[5] S.L. Schnee, N. A. Ridge, A.A. Goodman, G. L. Jason, APJ, 634, 442 (2005).

[6] X. Dupac, J.P. Bernard, N. Boudet, M. Giard, J.M. Lamarre, C. Meny, F. Pajot, I. Ristorcelli, G. Serra, B. Stepnik, J.P. Torre. A\&A, 404, L11 (2003).

[7] V. Konyves, Cs. Kiss, A. Moor, Z.T. Kiss, and L.V. Toth, A\&A, 463, 1227(2007).

[8] R.H. Hildebrand, Q.Jl.R.astr. obs.ser, 24, 267 (1983).

[9] D.O.S. Wood, P.C. Mayers \& D.A. Daughterty, the astrophysical journal supplement, 95, 457, (1994).

[10] K. Young, T.G. Phillips, G.R. Knapp, ApJ, 409, 725 (1993).

[11] B. Aryal, R. Weinberger,The Himalayan Physics, II, 5 (2011). 